\title{
DERIVA ORIGINADA POR DOS SISTEMAS DE APLICACIÓN DE PLAGUICIDAS EN HUERTOS DE CÍTRICOS
}

\author{
Rubén Collantes Veliz ${ }^{1}$, Casimiro Dias Gadanha Junior², Javier Alberto Vásquez-Castro³
}

\section{RESUMEN}

La deriva es el factor más importante que contribuye a la contaminación del ambiente durante la pulverización de plaguicidas. El presente trabajo tuvo por objetivo evaluar la deriva ocasionada por dos sistemas de aplicación de plaguicidas en huertos de cítricos. El experimento se realizó en un huerto de naranja de la variedad Valencia, ubicado en la hacienda Sertãozinho, Escola Superior de Agricultura Luiz de Queiroz, Piracicaba-SP. Se utilizó un pulverizador de chorro transportado con ventilador axial. Uno de los sistemas utilizó un prototipo de aplicación terrestre con atomizadores rotativos de discos que son mayormente empleados en aviación agrícola y el otro sistema utilizó las puntas hidráulicas de chorro cónico vacío. Para el tratamiento de los árboles se utilizó el insecticida organofosforado Metidation en la dosis de 180 g por hectárea. Para la evaluación de la endoderiva (pérdidas por escurrimiento) se colocaron láminas de vidrio y papeles hidrosensibles en el suelo, debajo de la copa de los árboles, mientras que para la evaluación de la exoderiva (pérdida por acción del viento) las superficies de colecta fueron colocadas sobre torres de madera a diferentes alturas en relación al suelo. Después de la pulverización, los papeles hidrosensibles fueron colectados y analizados a través de un sistema de análisis de imagen computarizado y las láminas de vidrio analizadas mediante técnica de cromatografía gaseosa. El sistema de aplicación equipado con atomizadores rotativos de discos causó menor deriva del plaguicida en relación al sistema equipado con puntas hidráulicas.

Palabras-clave: Tecnología de aplicación, puntas hidráulicas, atomizadores rotativos de disco, cromatografía, espectro de gotas.

\section{ABSTRACT \\ DRIFT PRODUCED BY TWO PESTICIDE APPLICATION SYSTEMS IN CITRUS ORCHARDS}

Drift is the most important factor contributing to environmental pollution during the spraying of pesticides. The objective of this work was to evaluate the drift caused by two pesticide spraying systems in citrus orchards. The experiment was conducted in an orchard of Valencia orange, located at the Sertãozinho farm, Escola Superior de Agricultura Luiz de Queiroz, Piracicaba-SP. An air-assisted sprayer with axial fan was used. One system used a prototype for terrestrial application with a rotary disc atomizer that is widely used in agricultural aviation, and the other system used hollow cone tip hydraulics. For treatment of the trees, the insecticide Metidation was used at the dose of $180 \mathrm{~g}$ per hectare. To assess runoff losses, glass slides and water-sensitive papers were placed on the ground below the canopy of the trees, while for the evaluation of drift losses the collection surfaces were placed on wooden towers at different heights above the ground. After spraying, the water-sensitive papers were collected and analyzed using a computerized image analysis system, e-Sprinkle (EMBRAPA, Sao Paulo, Brazil), and the glass slide analyzed by the gas chromatography technique. The application system equipped with rotary disc atomizers resulted in a lower pesticide drift in relation to the system that used hydraulic tips. Factors influencing drift are discussed in the present work.

Keywords: Application technology, hydraulic tips, rotary atomizers, chromatography, droplet spectrum.

\section{Recebido para publicação em 15/02/2012. Aprovado em 26/11/2012.}

1 - Ingeniero Agrícola, M.Sc. en Máquinas Agrícolas, Dep. de Engenharia de Biossistemas, ESALQ, SP, rdcollantes@hotmail.com

2 - Ingeniero Agrónomo, D.Sc. Profesor del Dep. de Engenharia de Biossistemas, ESALQ, SP, cdgadanh@usp.br

3 - Ingeniero Agrónomo, D.Sc. Profesor del Dep. de Entomología, Universidad Nacional Agraria La Molina, Lima-Perú, jaque@lamolina.edu.pe 


\section{INTRODUCCIÓN}

Los plaguicidas constituyen algunos de los insumos más importantes en sistemas modernos de producción agrícola y frecuentemente representan la primera medida de control en situaciones de irrupción de plagas. La utilización de éstos en programas de manejo integrado de plagas está condicionada a la evaluación de campo y disponibilidad de umbrales de acción (ZALOM, 2001). Sin embargo, a pesar de cumplir con estas condiciones, a menudo los plaguicidas son aplicados inadecuadamente, produciéndose fallas en el control de plagas. Este problema crea la necesidad de repetir las aplicaciones, aumentando el riesgo de intoxicación del agricultor, contaminación de los alimentos y el ambiente, y tornando el cultivo económicamente insostenible (VÁSQUEZ-CASTRO, 2004).

Las pérdidas que ocurren durante las aplicaciones de plaguicidas son originadas por un conjunto de causas. En las pulverizaciones con altos volúmenes muchas gotas caen entre las hojas de las plantas llegando al suelo, otras caen sobre las hojas, coalecen y escurren para el suelo, provocando así, la endoderiva. Por otro lado, en las pulverizaciones con bajos volúmenes, las gotas pequeñas que son más adecuadas para la penetración entre las hojas de la planta pueden ser llevadas por el viento para fuera del área tratada, ocasionando la exoderiva (MILLER, 1993). La contaminación del suelo ha causado gran variación en las poblaciones de organismos que no son objeto de control, principalmente aquellos que degradan la materia orgánica y mejoran la fertilidad (CHAIM, 2004). En muchos casos, la deriva puede ser la responsable por desequilibrios favorables al aparecimiento de nuevas plagas y enfermedades. El plaguicida puede ser llevado por el agua superficial o por el agua subterránea para ríos, lagos y océanos colocando en riesgo no solo a las poblaciones que viven en esos ecosistemas, sino también a las especies que utilizan esa agua para su sobrevivencia, como los animales y el hombre (VÁSQUEZ-CASTRO et al., 2011).

La búsqueda por la mejor forma de aplicación del plaguicida es tal vez más importante que los estudios de eficiencia biológica, pues este último representa apenas uno de los factores de interés en la protección de los cultivos. Un método de aplicación inadecuado ocasionará grandes pérdidas del plaguicida por efecto de la deriva, aumentando así, los problemas de contaminación del ambiente y colocando en riesgo la salud del agricultor, y la economía del productor.

En el sistema de aplicación, la "punta" de pulverización es el componente más importante, pues ella es la responsable por el caudal, generación y distribución de gotas que cargan el plaguicida hasta el blanco a ser controlado (VOLL et al., 2006). Las puntas hidráulicas son las más importantes en la aplicación de plaguicidas. En ellas, un líquido bajo presión es forzado a través de una pequeña abertura, de tal forma que el líquido se esparce, formando una lámina que posteriormente se desintegra en gotas de diferentes tamaños (VÁSQUEZ-CASTRO et al., 2007a). Otro tipo de sistema, aunque menos utilizado en aplicaciones terrestres es el atomizador rotativo de discos, el cual utiliza la energía centrífuga para la formación de gotas (LEFEBVRE, 1989). Diversos trabajos han demostrado las ventajas de estos atomizadores en relación a las puntas hidráulicas, entre las que se destacan, la homogeneidad en el espectro de gotas y la reducción en el volumen de aplicación, características que conducen a una mayor eficiencia biológica y reducción de los costos de operación (SANDER, 1991; VELIZ et al., 2010). El objetivo del presente trabajo fue evaluar la deriva ocasionada por dos sistemas de aplicación de plaguicidas en huertos de cítricos.

\section{MATERIALES Y MÉTODOS}

Los experimentos fueron conducidos en un huerto de naranjas de la variedad "Valencia" de 10 años de edad, con árboles distanciados a $6 \mathrm{~m}$ entre hileras y $3,5 \mathrm{~m}$ entre plantas, y con altura promedio de $4 \mathrm{~m}$, ubicado en la hacienda Sertãozinho, Escola Superior de Agricultura Luiz de Queiroz, PiracicabaSP. Se evaluaron cuatro árboles para cada sistema de aplicación, los cuales fueron seleccionados por sus semejanzas en la arquitectura de la copa a fin de estandarizar ese factor.

Las aplicaciones para ambos sistemas fueron realizadas con un pulverizador de tipo chorro transportado Arbus 2000, con deflector unilateral de tipo "camarón" con ventilador de $0,85 \mathrm{~m}$ de diámetro, el cual genera un flujo de aire de $12,6 \mathrm{~m}^{3} \cdot \mathrm{s}^{-1}$ según indicaciones del fabricante, tanque de fibra de vidrio de $2000 \mathrm{~L}$ de capacidad, bomba modelo JP150 de 150 L. $\mathrm{min}^{-1}$, comando convencional y filtro modelo FVS-200. Para accionar el pulverizador fue utilizado un tractor agrícola Massey Ferguson, modelo 5285 con motor Perkins, modelo 4001 de 85

\section{REVENG}

$$
\text { 129-137p. }
$$

ENGENHARIA NA AGRICULTURA, VIÇOSA - MG, V.21 N.2, MARÇO / Abril 2013 
$\mathrm{CV}$ de potencia en el motor.

Para el sistema de aplicación equipado con puntas hidráulicas se utilizó solamente el ramal inferior del deflector, provisto de 13 puntas de chorro cónico vacío modelo $\mathrm{AD} 3$ con difusor $\mathrm{AC} 45$. La presión del sistema fue de $827 \mathrm{kPa}$, según recomendación del fabricante. Para el sistema de aplicación equipado con atomizadores rotativos fue montada una estructura de fibra de vidrio con soportes para colocar cinco atomizadores rotativos, modelo TT-88B con las siguientes características técnicas: cuatro discos de plástico con periferia aserrada y diámetro de $0,088 \mathrm{~m}$ accionado por motor eléctrico, el cual es provisto de energía por la batería del tractor, disco de orifício D-4, velocidad de operación de 1,4 a 8,5 m.s. ${ }^{-1}$, velocidad de rotación de 2000 a $6000 \mathrm{rpm}$ con un peso aproximado de 1,67 kg. El control de las rotaciones de los discos del atomizador se realizó mediante un controlador de rotación marca Motron, modelo VV 8702, el cual es provisto de energía por la batería del tractor. La presión del sistema fue de $276 \mathrm{kPa}$.

Para la obtención del caudal real correspondiente para cada sistema de aplicación en las presiones de trabajo indicadas se recolectó durante un minuto el volumen en probeta de $2 \mathrm{~L}$ de capacidad. La velocidad de operación para el sistema de aplicación equipado con puntas hidráulicas fue de $0,83 \mathrm{~m} \cdot \mathrm{s}^{-1}$ correspondiente a la marcha del tractor $2^{\mathrm{a}}$ baja tortuga y para los atomizadores rotativos fue de 1,66 m.s. tractor $3^{\mathrm{a}}$ baja tortuga.

El volumen de caldo a utilizar en cada sistema de pulverización y la cantidad de insecticida (producto comercial) por hectárea fueron calculados a través de las fórmulas (1 y 2) propuestas por Ramos e Pio (2003).
$Q=\frac{600 \times q}{V \times e}$

Donde: $\mathbf{Q}$ es el volumen de aplicación (L.ha $\left.{ }^{-1}\right), \mathbf{q}$ es el caudal colectado de los atomizadores rotativos o puntas hidráulicas $\left(\right.$ L. $\left.\mathrm{min}^{-1}\right), \mathbf{V}$ es la velocidad del conjunto tractor-pulverizador $\left(\mathrm{km} \cdot \mathrm{h}^{-1}\right)$ y e es la distancia entre las hileras de los árboles (m).

$P c=\frac{D x C t}{Q}$

Donde: Pc es la cantidad de producto comercial (L), D es la dosis del producto $\left(\mathrm{L} . h a^{-1}\right), \mathbf{C t}$ es la capacidad del tanque (L) y $\mathbf{Q}$ es el volumen de aplicación (L.ha-1 ${ }^{-1}$.

Para el estudio de la deriva se utilizó el método propuesto por Salyani \& Farooq (2004) con algunas modificaciones. Para la evaluación de la endoderiva fueron colocadas cuatro láminas de vidrio $(0,1 \mathrm{~m}$ de largo, $0,05 \mathrm{~m}$ de ancho) y cuatro papeles hidrosensibles $(0,076 \mathrm{~m}$ de largo, $0,026 \mathrm{~m}$ de ancho) en el suelo, debajo de la copa de los árboles (Figura 1). Para la evaluación de la exoderiva fueron instalados siete postes de madera entre las hileras, cuatro de ellos ubicados en la dirección de los árboles y tres ubicados en la dirección del espacio entre los árboles (Figura 2). Las láminas de vidrio y los papeles hidrosensibles fueron instalados sobre los postes a 0,$50 ; 2,0 ; 3,5$; 5,0 y $5,50 \mathrm{~m}$ de altura en relación al suelo.
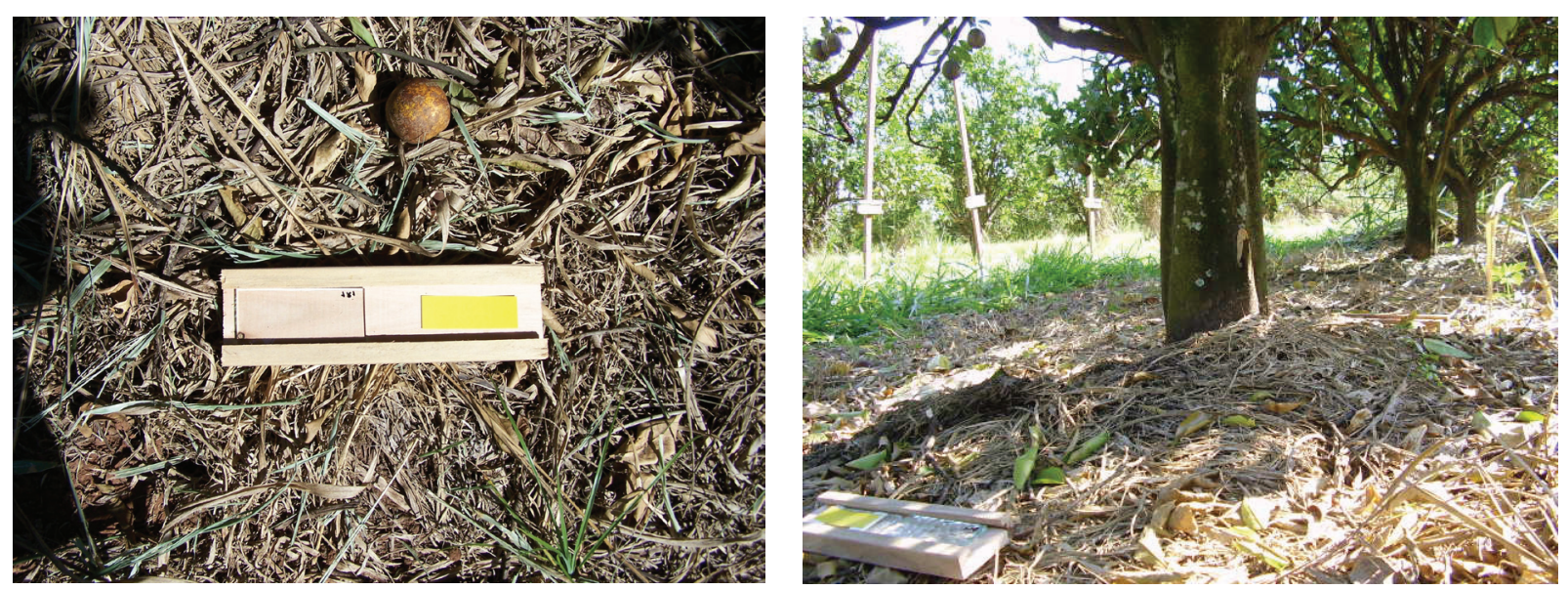

Figura 1. Láminas de vidrio y papeles hidrosensibles colocados debajo de la copa del árbol para el estudio de la endoderiva. 

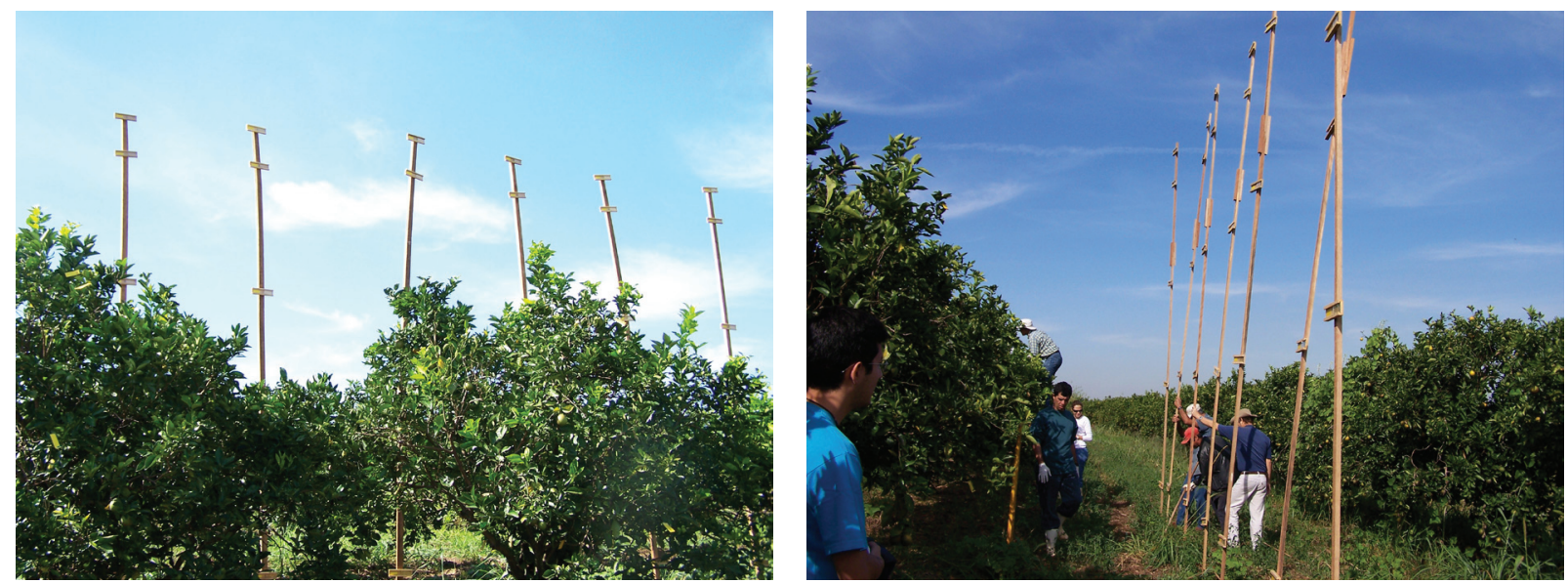

Figura 2. Postes de madera conteniendo láminas de vidrio y papeles hidrosensibles para el estudio de la exoderiva.

Para el tratamiento de los árboles se utilizó el insecticida Metidation (Supracid $400 \mathrm{CE}^{\circledR}$ ) a la dosis de 180 gr por hectárea. La aplicación se realizó por ambos lados de la hilera de árboles. La temperatura, humedad relativa y velocidad del viento durante las pulverizaciones fueron $29 \pm 1^{\circ} \mathrm{C}$, $58 \pm 2 \%$ y $1 \pm 0,2 \mathrm{~m} \cdot \mathrm{s}^{-1}$, respectivamente.

Para el estudio del espectro de gotas generado por ambos sistemas, los papeles hidrosensibles fueron escaneados y analizados a través de un sistema de análisis de imagen computarizado, e-Sprinkle (EMBRAPA, 2006).

Para el análisis del depósito del insecticida sobre las láminas de vidrio se utilizó el método propuesto por Vásquez-Castro et al. (2007b) con algunas modificaciones. Una lámina de vidrio fue colocada en frasco de $600 \mathrm{~mL}$. Se adicionaron $500 \mathrm{~mL}$ de acetato de etila, siendo posteriormente extraído el insecticida en Ultrason por $15 \mathrm{~min}$. Después de esta operación fueron transferidas alícuotas de $40 \mathrm{~mL}$ para tubos de ensayo de $50 \mathrm{~mL}$, siendo, en seguida, adicionados $50 \mu \mathrm{L}$ de dodecano. Los extractos fueron evaporados hasta obtener $1 \mathrm{~mL}$ en TurboVap, en baño-maria a $40^{\circ} \mathrm{C}$, con el auxilio de aire circulante, previamente seco en filtro de sílica-gel desecante azul, seguido de inyección en el sistema cromatográfico.

Lasmuestrasfueronanalizadasporcromatografía en fase gaseosa, usándose cromatógrafo a gas, Varian, modelo Star 3400X, equipado con detector de ionización de llama y columna cromatográfica capilar Paropak con inyecciones hechas en modo "splitless". Las condiciones de operación del cromatógrafo fueron: temperatura de la columna
$=120{ }^{\circ} \mathrm{C}$ (partida) por un minuto; después a 280 ${ }^{\circ} \mathrm{C}$ (rampa de $30{ }^{\circ} \mathrm{C} \cdot \mathrm{min}^{-1}$ ), donde permaneció por periodo de 5 minutos; temperatura del inyector $=$ $220{ }^{\circ} \mathrm{C}$; temperatura del detector $=300{ }^{\circ} \mathrm{C}$; tiempo de purga $=1$ minuto; flujo de gases $\left(\mathrm{mL} \cdot \mathrm{min}^{-1}\right)$ : $\mathrm{He}($ arrastre $)=5 ; \mathrm{N}_{2}($ make up $)=20$. En estas condiciones, el tiempo de retención del metidation fue $8 \mathrm{~min} 30 \mathrm{~s}$, aproximadamente. Los depósitos fueron calculados usando el software ChromQuest versión 4,0, a partir de la comparación de las alturas de los picos cromatográficos de las muestras con las alturas de los picos cromatográficos de los padrones analíticos correspondientes.

Para el análisis estadístico de los datos referentes a la endoderiva, fueron calculados los promedios de las cuatro medidas realizadas debajo de la copa de cada árbol, considerando así, cada árbol como una parcela experimental. Para comparar los dos sistemas de aplicación fue efectuada la prueba de t para comparación de promedios considerando variancias iguales o desiguales, conforme el caso. La evaluación de la igualdad de las variancias fue realizada por medio de la prueba de F (COSTA NETO, 1998). El análisis estadístico fue realizado a través del procedimiento de TTEST del SAS (1999).

Para el análisis de los datos referentes a la exoderiva, cada torre fue considerada como una parcela experimental, mientras que las alturas fueron consideradas como subparcelas. Fueron calculados los promedios de las dos pasadas del equipo pulverizador para cada poste y para cada altura. A partir de los promedios fue realizado el análisis de datos utilizando el modelo matemático para el delineamiento enteramente al azar con

\section{2 \\ REVENG 129-137p.}


tratamientos dispuestos en el esquema de parcelas subdivididas (split-plot). El efecto del sistema de aplicación fue considerado como asociado a la parcela, mientras que el efecto de la altura fue considerada como relacionada a la subparcela. Además de eso, como había posibilidad de la existencia de heterogeneidad de variancias relacionada a los sistemas de aplicación, fueron ajustados dos modelos, uno considerando homogeneidad de variancias entre los sistemas de aplicación y el otro considerando heterogeneidad de variancias. La elección de uno de los dos modelos para finalizar el análisis fue en base al Criterio de Información de Akaike (SAS, 1999). El análisis fue realizado a través del procedimiento MIXED del SAS (1999).

\section{RESULTADOS Y DISCUSIÓN}

El caudal promedio del atomizador rotativo de discos fue 2,15 L. $\mathrm{min}^{-1}$, de esa forma, el caudal total del sistema (5 atomizadores rotativos) fue 10,75 L. $\mathrm{min}^{-1}$. La velocidad de operación del conjunto tractor-pulverizador fue 1,67 m.s. ${ }^{-1}$. Como se utilizó apenas un lado del pulverizador para la aplicación, la distancia entre las hileras de árboles fue divido entre 2 a fin de calcular el volumen de caldo, cuyo valor según la Fórmula 1 fue de 360 L.ha ${ }^{-1}$. De acuerdo a la recomendación del fabricante del insecticida, la formulación comercial es utilizada a la concentración de $0,125 \%$. De esa forma fueron utilizados $500 \mathrm{~L}$ de agua y $0,625 \mathrm{~L}$ del producto comercial para preparar el caldo insecticida.

Con el objetivo de utilizar la misma dosis del insecticida en ambos sistemas de aplicación fue despejada la Fórmula 2, cuyo valor fue de $0,45 \mathrm{~L}^{\text {.ha }}{ }^{-}$ ${ }^{1}$ del producto comercial (180 g de Metidation por hectárea). El caudal promedio de la punta hidráulica fue $1,7 \mathrm{~L} \cdot \mathrm{min}^{-1}$, de esa forma, el caudal total del sistema (13 puntas hidráulicas) fue 22,1 L. min ${ }^{-1}$. La velocidad de operación del conjunto tractorpulverizador fue estimada en $0,83 \mathrm{~m} \cdot \mathrm{s}^{-1}$ (Fórmula 1) a fin de aplicar el volumen de caldo recomendado por el fabricante del insecticida de 1500 L.ha ${ }^{-1}$.

Esos resultados muestran que el sistema de aplicación equipado con atomizadores rotativos de disco utilizó apenas un cuarto del volumen de caldo utilizado por los sistemas de aplicación tradicionales (puntas hidráulicas). Debido al comportamiento variable de las plagas en que algunas se localizan en la parte externa de la copa de los árboles y otras en la parte interna, y a la gran variabilidad en la arquitectura de las copas, las pulverizaciones generalmente son realizadas con volúmenes de caldo elevados en la errónea creencia de que así, se aumentará la eficacia del producto químico. Por otro lado, el alto costo de operación de las aplicaciones de plaguicidas asociado al requerimiento de las empresas agrícolas de reducir sus costos de producción ha creado la imperiosa necesidad de mejorar la eficiencia de los sistemas de aplicación tradicionales.

El Cuadro 1 muestra los promedios y desviaciones estándar de las variables relacionadas a la endoderiva. Para las variables: depósito, diámetro mediano volumétrico y amplitud relativa la prueba de $\mathrm{F}$ detectó diferencias significativas $(P \leq 0,05)$ entre las variancias de los dos sistemas de aplicación. A pesar que el sistema de aplicación con atomizadores rotativos de disco presentó valores de depósito en la lámina de vidrio y de diámetro mediano volumétrico inferiores al sistema equipado con puntas hidráulicas, esos valores no difirieron significativamente $(P>0,05)$ entre si. Se observa que la variabilidad de los promedios es mayor en las puntas hidráulicas en relación a los atomizadores rotativos de disco, siendo verificado a través de la prueba de $\mathrm{F}(P \leq 0,05)$ para comparación de variancias.

El volumen de aplicación depende del tipo de tratamiento que se desea ejecutar, aunque presenta una fuerte relación con el tamaño de gotas producidas por los sistemas de pulverización, los cuales determinan la distribución del plaguicida en el "blanco". Poca atención se ha dado al tamaño de las gotas y una gran variedad de puntas de pulverización ha sido utilizada a lo largo de los años. La mayoría de las puntas produce un espectro de gotas de tamaños variados y, en muchos casos, las gotas grandes impactan contra las hojas externas de los árboles y no consiguen alcanzar la parte más interna de la copa (VELIZ et al., 2010). Ese depósito externo puede darse en tal intensidad que acaba escurriendo para el suelo.

Actualmente, las recomendaciones contenidas en las etiquetas de los plaguicidas dejan a criterio del aplicador la elección del volumen de aplicación. Para el tratamiento de árboles de cítricos, las recomendaciones dan opciones de 1500 a más de 3000 L.ha $^{-1}$ sin tener en consideración el problema antes expuesto. 
Cuadro 1. Promedios y desviaciones estándar de las variables relacionadas a la endoderiva y nivel descriptivo de probabilidad de la prueba de $\mathrm{t}$

\begin{tabular}{cccc}
\hline \multirow{2}{*}{ Variables } & \multicolumn{2}{c}{ Sistema de aplicación } & \multirow{2}{*}{$\operatorname{Pr}>\mathrm{t}$} \\
\cline { 2 - 3 } & Punta hidráulica & $\begin{array}{c}\text { Atomizador rotativo de } \\
\text { discos }\end{array}$ & \\
\hline Depósito en la lámina de vidrio $\left(\mathrm{ng} . \mathrm{cm}^{-2}\right)$ & $25,23 \pm 10,35$ & $3,43 \pm 0,76$ & 0,1255 \\
Diámetro mediano volumétrico DMV $(\mu \mathrm{m})$ & $742,09 \pm 199,5$ & $261,88 \pm 15,92$ & 0,0948 \\
Amplitud relativa & $1,24 \pm 0,03$ & $1,75 \pm 0,14$ & 0,0300 \\
Densidad de gota DG (Número de gotas.cm $\left.\mathrm{cm}^{-2}\right)$ & $218,99 \pm 29,80$ & $301,82 \pm 21,38$ & $0,0647^{*}$ \\
\hline
\end{tabular}

* Test t para comparación de promedios con variancias iguales.

Cuadro 2. Niveles descriptivos de probabilidad de la prueba de F, para las variables relacionadas con la exoderiva

\begin{tabular}{ccccc}
\hline Causas de variación & $\begin{array}{c}\text { Depósito en la } \\
\text { lámina de vidrio* }\end{array}$ & DMV $^{* *}$ & $\begin{array}{c}\text { Amplitud } \\
\text { relativa** }\end{array}$ & $\begin{array}{c}\text { Densidad de } \\
\text { gota** }\end{array}$ \\
\hline Sistema de aplicación & \multicolumn{2}{c}{ Postes en la dirección de los árboles } & & \\
Altura & 0,0036 & 0,0007 & 0,0053 & 0,0012 \\
Altura $\times$ Sist. aplicación & $<0,0001$ & 0,0008 & 0,0002 & $<0,0001$ \\
& $<0,0001$ & 0,0129 & 0,0002 & 0,0039 \\
Sistema de aplicación & Postes en la dirección del espacio entre árboles & \\
Altura & 0,0247 & 0,5888 & 0,1052 & 0,0187 \\
Altura $\times$ Sist. aplicación & 0,0174 & 0,3137 & 0,0232 & 0,3658 \\
\hline
\end{tabular}

* Modelo con heterogeneidad de variancias entre los sistemas de aplicación.

** Modelo con heterogeneidad de variancias entre los sistemas de aplicación.

En el Cuadro 2 son presentados los niveles descriptivos de probabilidad de la prueba de $\mathrm{F}$ para las variables relacionadas a la exoderiva. El análisis del depósito en la lámina de vidrio fue realizado considerando variancias diferentes entre los sistemas de aplicación, con base en el valor del Criterio de Información de Akaike. Para las otras tres variables (DMV, amplitud relativa y densidad de gota) no hubo necesidad de realizar el análisis considerando homogeneidad de variancias. En el caso de las variables medidas en los postes ubicados en la dirección de los árboles, hubo efecto significativo de todos los factores evaluados $(P \leq 0,05)$, en tanto que, para las variables medidas en los postes ubicados en la dirección del espacio entre los árboles, hubo efecto significativo de todos los factores para el depósito en la lámina de vidrio, de la altura para la amplitud relativa y del sistema de aplicación para la densidad de gota. Ese resultado indica que el efecto del sistema de aplicación depende de la altura, y vice-versa, para todas las variables medidas en los postes localizados en la dirección de los árboles y también para el depósito en la lámina de vidrio cuando medido en los postes localizados en la dirección del espacio entre los árboles.

Debido a esa coyuntura se optó por presentar los promedios de las variables para la interacción entre altura y sistema de aplicación (Cuadro 3). Las diferencias entre los sistemas de aplicación fueron más evidentes en las extremidades de los postes $(0,5 ; 5,0$ y $5,5 \mathrm{~m})$, mientras que en las alturas intermedias $(2,0$ y $3,0 \mathrm{~m})$ apenas hubo diferencia significativa entre los sistemas de aplicación para la amplitud relativa medida en los postes localizados en la dirección de los árboles. En prácticamente todos los casos en que hubo efecto significativo del sistema de aplicación, el mayor valor promedio fue observado en el sistema de aplicación equipado con puntas hidráulicas. Por otro lado, no hubo

\section{REVENG}


efecto significativo de la altura dentro del sistema de aplicación equipado con atomizadores rotativos de disco, excepto para la amplitud relativa medida en los postes localizados en la dirección del espacio entre los árboles. En el caso del sistema equipado con boquillas hidráulicas, hubo efecto significativo para la altura en todos los casos, excepto para el DMV y la densidad de gota, para las medidas realizadas en los postes localizados en la dirección del espacio entre los árboles.

Los mayores depósitos en las extremidades de los postes indican que la exoderiva del spray ocurre principalmente por debajo y por encima de la copa de los árboles. Esto se debe a que el flujo de aire del pulverizador sigue la trayectoria de menor resistencia a su desplazamiento(CROSS etal.,2001; FAROOQ; SALYANI, 2004; VILLALBA; HETZ, 2010). Por otro lado, a pesar de que el sistema de pulverización equipado con atomizadores rotativos de disco genera gotas pequeñas, las que a su vez son más susceptibles a la exoderiva, este sistema ocasionó menor contaminación del ambiente, pues las gotas pequeñas son también las más adecuadas para la penetración entre las hojas de la copa del árbol, aumentando así, el depósito del plaguicida en el "blanco" deseado.

Cuadro 3. Promedios y desviación estándar de las variables relacionadas a la exoderiva en función del sistema de aplicación y de la altura

\begin{tabular}{|c|c|c|c|c|c|}
\hline \multirow[t]{2}{*}{ Sistema de aplicación } & \multicolumn{5}{|c|}{ Altura (m) } \\
\hline & 0,5 & 2,0 & 3,0 & 5,0 & 5,5 \\
\hline \multicolumn{6}{|c|}{ POSTES EN LA DIRECCION DE LOS ÁRBOLES } \\
\hline \multicolumn{6}{|c|}{ Depósito en la lámina de vidrio (ng. $\mathrm{cm}^{-2}$ ) } \\
\hline Atomizador rotativo & $0,374 \pm 1,426^{b}$ & $0,000 \pm 1,426$ & $0,350 \pm 1,426$ & $0,510 \pm 1,426^{b}$ & $0,040 \pm 1,426^{\mathrm{b}}$ \\
\hline Punta hidráulica* & $22,03 \pm 2,796^{\text {a }}$ & $1,692 \pm 2,796$ & $6,709 \pm 2,796$ & $16,412 \pm 2,796^{\mathrm{a}}$ & $13,765 \pm 2,796^{\mathrm{a}}$ \\
\hline \multicolumn{6}{|c|}{ Diámetro Mediano Volumétrico DMV $(\mu \mathrm{m})$} \\
\hline Atomizador rotativo & $174,90 \pm 73,93^{b}$ & $85,10 \pm 73,93$ & $129,73 \pm 73,93$ & $155,20 \pm 73,93^{\mathrm{b}}$ & $166,69 \pm 73,93^{b}$ \\
\hline Punta hidráulica* & $757,25 \pm 73,93^{\text {a }}$ & $194,71 \pm 73,93$ & $212,01 \pm 73,93$ & $453,66 \pm 73,93^{\text {a }}$ & $570,45 \pm 73,93^{\text {a }}$ \\
\hline \multicolumn{6}{|c|}{ Amplitud relativa } \\
\hline Atomizador rotativo & $0,550 \pm 0,069^{\mathrm{b}}$ & $0,475 \pm 0,069^{\mathrm{b}}$ & $0,600 \pm 0,069$ & $0,525 \pm 0,069$ & $0,488 \pm 0,069$ \\
\hline Punta hidráulica* & $1,200 \pm 0,069^{\mathrm{a}}$ & $0,700 \pm 0,069^{\mathrm{a}}$ & $0,475 \pm 0,069$ & $0,688 \pm 0,069$ & $0,600 \pm 0,069$ \\
\hline \multicolumn{6}{|c|}{ Densidad de gota (Número de gotas.cm²) } \\
\hline Atomizador rotativo & $56,65 \pm 16,72^{b}$ & $7,90 \pm 16,72$ & $42,75 \pm 16,72$ & $42,45 \pm 16,72^{b}$ & $25,45 \pm 16,72^{b}$ \\
\hline Punta hidráulica* & $212,10 \pm 16,72^{a}$ & $27,66 \pm 16,72$ & $86,28 \pm 16,72$ & $95,75 \pm 16,72^{a}$ & $70,04 \pm 16,72^{\mathrm{a}}$ \\
\hline
\end{tabular}

\section{POSTES EN LA DIRECCIÓN DEL ESPACIO ENTRE ÁRBOLES}

\begin{tabular}{|c|c|c|c|c|c|}
\hline \\
\hline Atomizador rotativo & $0,338 \pm 3,125^{b}$ & $1,097 \pm 3,467$ & $0,307 \pm 3,125$ & $0,080 \pm 3,125$ & $0,266 \pm 3,125^{b}$ \\
\hline Punta hidráulica* & $25,978 \pm 3,467^{\text {a }}$ & $4,799 \pm 3,125$ & $4,385 \pm 3,467$ & $6,290 \pm 3,467$ & $11,565 \pm 3,467^{\mathrm{a}}$ \\
\hline \multicolumn{6}{|c|}{ Diámetro Mediano Volumétrico DMV $(\mu \mathrm{m})$} \\
\hline Atomizador rotativo & $164,6 \pm 281,9$ & $219,3 \pm 281,9$ & $87,5 \pm 281,9$ & $145,8 \pm 281,9$ & $162,8 \pm 281,9$ \\
\hline Punta hidráulica & $1066,9 \pm 281,9$ & $978,1 \pm 281,9$ & $232,5 \pm 281,9$ & $291,4 \pm 281,9$ & $251,7 \pm 281,9$ \\
\hline \multicolumn{6}{|c|}{ Amplitud relativa } \\
\hline Atomizador rotativo* & $0,633 \pm 0,131$ & $0,400 \pm 0,131$ & $1,000 \pm 0,131$ & $0,433 \pm 0,131$ & $0,350 \pm 0,131$ \\
\hline Punta hidráulica* & $1,050 \pm 0,131$ & $0,567 \pm 0,131$ & $0,683 \pm 0,131$ & $0,767 \pm 0,131$ & $0,617 \pm 0,131$ \\
\hline \multicolumn{6}{|c|}{ Densidad de gota (Número de gotas. $\mathrm{cm}^{-2}$ ) } \\
\hline Atomizador rotativo & $54,67 \pm 40,37^{\mathrm{b}}$ & $54,95 \pm 40,37$ & $33,15 \pm 40,37$ & $34,17 \pm 40,37^{\text {a }}$ & $20,67 \pm 40,37^{\mathrm{b}}$ \\
\hline Punta Hidráulica & $198,92 \pm 40,37^{\text {a }}$ & $78,60 \pm 40,37$ & $77,23 \pm 40,37$ & $192,07 \pm 40,37^{b}$ & $186,30 \pm 40,37^{\mathrm{a}}$ \\
\hline
\end{tabular}

Medias seguidas por letras distintas en las columnas difieren significativamente por el test $\mathrm{F}(\mathrm{p} \leq 0,05)$;

* Hubo efecto significativo de la altura dentro del sistema de aplicación por el test $\mathrm{F}(\mathrm{p} \leq 0,05)$. 


\section{CONCLUSIÓN}

- El sistema de aplicación equipado con atomizadores rotativos de disco produce menores pérdidas por deriva que aquel equipado con puntas hidráulicas. Se recomienda así, la utilización de esos atomizadores para minimizar la deriva de plaguicidas en huertos de cítricos.

\section{AGRADECIMIENTOS}

A Gilberto Casadei de Baptista y Luiz Roberto Pimentel Trevizan por la orientación durante los análisis cromatográficos y Arlei Coldebella por su orientación en el análisis estadístico.

\section{REFERENCIAS BIBLIOGRÁFICAS}

CHAIM, A. Tecnologia de aplicação de agrotóxicos: fatores que afetam a eficiência e o impacto ambiental. In: SILVA, C.M.; FAY, E.F. (eds.). Agrotóxicos e ambiente. Brasilia, DF: Embrapa, 2004, p.289-317.

COSTA NETO, P.L.O. Estatística. São Paulo: Edgard Blücher, 1998. 264 p.

CROSS, J.V.; WALKLATE, P.J.; MURRAY, R.A.; RICHARDSON, G.M. Spray deposits and losses in differents sized apple trees from an axial fan orchard sprayer: 2. Effects of spray quality. Crop Protection, v.20, p. 333-343, 2001.

EMPRESA BRASILEIRA DE PESQUISA AGROPECUARIA. e-Sprinkle Guia de Usuario. SP: EMBRAPA Inc. 1 CD-ROM, 2006.

FAROOQ, M.; SALYANI, M. Modeling of spray penetration and deposition on citrus tree canopies. Transactions of the ASAE, v.47, p. 619-627, 2004.

LEFEBVRE, A.H. Atomization and sprays. New York: Taylor \& Francis, 1989. 421 p.

MILLER, P.C,H. Spray drift and its measurement. In: MATTHEWS, G.A.; HISLOP, E.C. (eds.). Application technology for crop protection. Wallingford: Cab International, 1993, p.101-122.
RAMOS, H.H.; PIO, L.C. Tecnologia de aplicação de produtos fitossanitários. In: ZAMBOLIM, L.; CONCEIÇÃO, M.Z.; SANTIAGO, T. (eds.). $\mathrm{O}$ que engenheiros agrônomos devem saber para orientar o uso de produtos fitossanitários. Viçosa: ANDEF, 2003, p.133-201.

SALYANI, M.; FAROOQ, M. Drift potential of citrus of air-carrier sprayers. Proceeding of Florida State Horticultural Society, v.111, p.130135, 2004.

SANDER, T.P.Y. Development and evaluation of rotary cage atomizer conversion for orchard sprayer.

Air Assisted Spraying in Crop Protection, v.46, p.203-210, 1991.

SAS Institute. SAS/STAT User's Guide 8.0. Cary: SAS Institute Inc. 1 CD-ROM, 1999.

VÁSQUEZ-CASTRO, J.A. Propuesta de manejo integrado de plagas del algodonero en el valle del Santa, Ancash, Perú. Revista Peruana de Entomología, v.44, p.119-124, 2004.

VÁSQUEZ-CASTRO, J.A.; DI LORENZI, G.; MONTES, F. Manual de Aplicaciones. Lima: Procitrus, 2011. 81p.

VÁSQUEZ-CASTRO, J.A.; BAPTISTA, G.C.; GADANHA JR., C.D.; TREVIZAN, L.R.P. Influence of emulsifiable concentrate formulation on the physical properties of the fluid, spray characteristics, and insecticide deposits on stored grains. Journal of Agricultural and Food Chemistry, v.55, p.3529-3534, 2007a.

VÁSQUEZ-CASTRO, J.A.; BAPTISTA, G.C.; GADANHA JR., C.D.; TREVIZAN, L.R.P. A system to evaluate the performance of hydraulic nozzles used in stored grain protection trials. Scientia Agricola, v.64, p.563-568, 2007 b.

VELIZ, R.C.; GADANHA JR., C.D.; VÁSQUEZCASTRO, J.A. Eficiencia de dos sistemas para la aspersión de plaguicidas en árboles de cítricos. Revista Colombiana de Entomología, v.36, p.217-222, 2010. 
VILLALBA, J.; HETZ, E. Deriva de productos agroquímicos - Efecto de las condiciones ambientales. In: MAGDALENA, J.C.; CASTILLO, H.B.; DI PRINZIO, A.; HOMER B.I.; VILLALBA, J. (eds.). Tecnología de aplicación de agroquímicos. Alto Valle: INTA, 2010, p.45-54.

VOLL, C.E.; VÁSQUEZ-CASTRO, J.A.; GADANHA JR., C.D.; LINO, P.L. Distribución volumétrica de la boquilla de pulverización XR11003. Revista Peruana de Entomología, v.45, p.95-99, 2006.

ZALOM, F.G. Pesticide use practices in integrated pest management. In: KRIEGER, R.I. (ed.). Handbook of pesticide toxicology. 2 ed. Toronto: Academic Press, 2001, v.1, p.275-283. 